\title{
Triple Hormonal Blockade (ADT3): A Patient's Perspective
}

\section{Charles Maack*}

US Too International Inc, USA

\section{*Corresponding author: \\ Charles Maack}

US Too International Inc,

5003 Fairview Ave, Downers Grove, IL 60515, USA

Received : January 23, 2019

Published : January 30, 2019

to enhance the effectiveness of the chemotherapy medication docetaxel/Taxotere.

Triple-hormonal blockade/androgen deprivation therapy (ADT3) includes the prescribing of a GnRH agonist or antagonist (I use LHRH and GnRH in the following but in reality, either is appropriate for agonists) to shut down testicular testosterone production; an antiandrogen to block testosterone access to the cancer cell nucleus; and a 5Alpha Reductase (5AR) inhibitor to prevent any testosterone that might access the cancer cell nucleus from converting to dihydrotestosterone/DHT. $\mathrm{DHT}$ is a 5 times more powerful stimulant than testosterone to prostate cancer cell growth and proliferation.

The following is a reasonable description of the importance of these medications that should be employed - and why - to manage and control prostate cancer cell development either as primary therapy for advanced disease, in company with other treatment options, or following earlier treatment protocols that have failed. [http://tinyurl.com/optfxp8]

Note: Since there can be cardiac risk, and adverse effects on diabetics and those susceptible to stroke associated with the administration of ADT medications, it is important the prescribing physician first perform necessary testing to rule out this possibility. According to this report [http://tinyurl. com/73rhbgv], the addition of androgen deprivation therapy in men with clinically localized prostate cancer was not associated with increased cardiovascular mortality. However, cardiac risk is of more concern for those patients, who, when the administration of a $\mathrm{GnRH}$ agonist or antagonist is being considered, are already experiencing cardiovascular disease, diabetes, susceptible to stroke, or are 70 years of age or older. A report in February 2014 titled, "Potential 50\% reduction in cardiac events for prostate cancer patients when treated with degarelix" suggests that for patients with known cardiovascular disease/CVD, rather than prescribing a $\mathrm{GnRH}$ "agonist" such as Lupron, Zoladex, Trelstar, Eligard, or Vantas, instead prescribe the GnRH "antagonist" Firmagon/degarelix. Should you be such a patient, certainly bring this to the attention of 
your treating physician hopefully before beginning androgen deprivation therapy. [See: https://www.ferring.com/en/media/press-releases/firmagon-5feb14.aspx].

For patients who had congestive heart failure or a previous myocardial infarction prior to the consideration of prescribing ADT medications, it is extremely important to make your prostate cancer treating physician aware!

Additionally, all patients should have their liver functions tested before the prescribing of either antiandrogens or 5Alpha Reductase (5AR) inhibitors since these medications can enhance damage to liver already experiencing problems.

GnRH agonists or antagonist, antiandrogens, and 5alpha reductase (5AR) inhibitor each have absolutely different, but important, individual functions in ADT.

As mentioned previously, GnRH agonists include Lupron, Eligard, Trelstar, Zoladex, or Vantas, or the GnRH antagonist is Firmagon/degarelix; antiandrogens include Casodex/bicalutamide (with the generic bicalutamide now more often prescribed), Eulexin/flutamide, or Nilandron/nilutamide; and 5AR inhibitors are either Avodart/dutasteride or Proscar/finasteride.

Lupron (and other GnRH agonists), as well as the GnRH antagonist Firmagon, shut down a complex system of production of testosterone via hypothalamus/pituitary/luteinizing hormone (LH)/Leydig Cell stimulation in the testicles.

Take special note: Patients being prescribed androgen deprivation who have been diagnosed with aggressive, higher grade prostate cancer, as well as if known metastasis is present, would be better served to be prescribed the antagonist Firmagon/degarelix, at least as their initial medication. The reasoning is that Firmagon shuts down testosterone nearly immediately without causing the surge in testosterone that occurs with GnRH agonists. That surge, also known as "flare," if prescribed an agonist, and particularly if not preceded by beginning an antiandrogen at least a week prior to administration, can be extremely painful to metastatic sites and in some cases dangerous. The "do no harm" is important in this regard.

Also important to be considered if experiencing high-extent metastatic prostate cancer, is beginning the chemotherapy medication docetaxel/Taxotere in company with the foregoing ADT medications. High-extent - meaning the disease had spread to major organs such as the liver, had a spread resulting in four or more bone lesions, or both (take note, above, if already experiencing liver issues) - In this case of "high-extent" metastasis, docetaxel/Taxotere should be administered every three weeks for a total of 18 weeks after which the docetaxel/ Taxotere can be stopped and the ADT medications continued. According to the following paper with this procedure in trials, "A significant improvement in the overall survival was noted favoring the participants who had received docetaxel chemotherapy in addition to the ADT compared to the ADT alone." This addition of docetaxel/Taxotere is not considered necessary for those patients with low-extent metastasis. [See http:// www.nih.gov/news/health/dec2013/nci-05.htm]

A video presentation by renowned Medical Oncologist Mayers CE, [http://tinyurl.com/qgs7sc5] a specialist specifically in research and treatment of recurring and advanced prostate cancer, that supports the consideration of moving to this chemotherapy agent early on.

Medical oncologist Strum S, specializing specifically in the treatment of recurring and/or advanced/high grade prostate cancer since 1983: In the case of those of you who have been on an LHRH agonist (or GnRH antagonist) for a long time (near or over 24 months), simply measure the testosterone ( $T$ ) using the LC/MS/MS methodology of testosterone testing. If your $\mathrm{T}$ is $<32 \mathrm{ng} / \mathrm{dl}(<1.11 \mathrm{nmol} / \mathrm{L})$ then you are fine, and if lower that might even be better. This gives you the opportunity to take a break from the agonist or antagonist. Repeat the same testosterone measurement (using the LC/MS/MS methodology) monthly. You can expect an upward trend of testosterone, but if there is as well an increase in PSA, then recovery of the receptors in the hypothalamic-pituitary axis (HPA) is occurring and time to reinitiate the agonist or antagonist injection.

LHRH agonists or the GnRH antagonist DO NOT shut down the production of testosterone metabolized from androgen precursors in the adrenal glands. And the production of those androgen precursors (that convert to testosterone) is even enhanced when LH/leydig cell production via the testicles is stopped. So, here you still have adrenal gland androgen/testosterone having access to cancer cell development.

Casodex (more often prescribed in its generic form, bicalutamide) (and other antiandrogens) has a structure similar to testosterone. It works by preventing testosterone from attaching (binding) to and activating androgen receptors on prostate cancer cells. Without testosterone the cancer cells either grow more slowly or stop growing altogether. The cancer tumor may shrink in size as a result. Though this medication is prescribed to hopefully block activation of all androgen recep- 
tors in all cancer cells so that androgen/testosterone cannot access those cancer cells, it is unlikely the multitude of androgen receptors are all blocked; those not blocked still open access of adrenal gland androgen/testosterone to the cancer cell nucleus despite agonists or antagonist hopefully shutting down all testicular testosterone production. This being the case, in the cancer cell nucleus are Type I and Type II enzymes known as 5Alpha Reductase (5AR). When androgen/testosterone comes in contact with these 5AR enzymes, it converts to an up to a five times or more powerful stimulant to cancer cell growth, dihydrotestosterone (DHT). Obviously, this should be prevented. And remember, LHRH agonists or GnRH antagonist do NOT stop adrenal gland androgen/testosterone production and as earlier noted, it is unlikely all androgen receptors are blocked by an antiandrogen.

So, we now want to inhibit these 5AR enzymes from converting androgen/testosterone from any source to the more powerful cancer stimulant, DHT. We do that with a 5AR"inhibitor." And those inhibitors are primarily dutasteride/Avodart or finasteride/Proscar. And since Proscar only inhibits Type II enzymes, Avodart is the better inhibitor because it inhibits both Type I and Type II enzymes. A randomized, double-blind, placebo-controlled trial has confirmed the importance of including dutasteride/Avodart in androgen deprivation therapy. [See: http://www.ncbi.nlm.nih.gov/pubmed/23176897]. Schröder F, et al. (2013), concludes that Dutasteride delayed the biochemical progression of PCa in patients with biochemical failure after radical therapy for clinically localized disease. The safety and tolerability of dutasteride were generally consistent with previous experience.

For an in-depth understanding on the role of dutasteride/ Avodart in Androgen Deprivation Therapy, take the IMPORTANT time to view and listen to this presentation by Myers CE and take special note of the importance to control "dihydrotestosterone/DHT." It is very important that when prescribing androgen deprivation therapy that the patient's dihydrotestosterone level be monitored as well as PSA, testosterone, and Prolactin level. Also take special note that when Zytiga/abiraterone acetate shows failure, dutasteride/Avodart has been found to continue effectiveness in controlling testosterone from converting to dihydrotestosterone: [https://askdrmyers. wordpress.com/2013/10/15/avodart-in-depth/]

From Medical Oncologist Mark Scholz, specializing specifically in research and treatment of recurring and advanced prostate cancer regarding the importance of dutasteride/Avodart, or alternatively finasteride/Proscar: [http://prostatesnatchers. blogspot.com/2014/10/avodart-proscar.html]

"Blog" by Myers CE, regarding where Avodart could be important as a monotherapy when a patient experiences PSA elevation post-surgical removal as evidence of prostate cancer recurrence. According to Dr. Myers, Avodart monotherapy could delay cancer progression as well as avoid the many side effects that can accompany, for example, salvage radiation, or even the prescribing of androgen deprivation medications (LHRH agonists or GnRH antagonist, and antiandrogens): http://tinyurl.com/owtlsss

Modern thinking physicians who have followed many persons on LHRH agonists or the GnRH antagonist Firmagon/ degarelix, have determined how detrimental it is for patients to be on continuous androgen/testosterone deprivation; they have determined that intermittent androgen deprivation (IAD) (stopping use of the GnRH agonist or antagonist and the antiandrogen) can be as beneficial as continuous therapy as long as the individual has been able to obtain and maintain for 1 year a PSA level that is "undetectable" or $<0.05 \mathrm{ng} / \mathrm{ml}$ and a "castrate" testosterone $(T)$ level $<32$ ng/dl (but preferably $<20 \mathrm{ng} / \mathrm{dl}$ ) before considering IAD. Dr. Strum also provides this important consideration that I made note above and will repeat here: In the case of those of you who have been on LHRH agonists for a long time (approaching or over 24 months), simply measure the testosterone using the LC/MS/MS methodology of testosterone testing. If your $\mathrm{T}$ is $<32 \mathrm{ng} / \mathrm{dl}(<1.11$ $\mathrm{nmol} / \mathrm{L}$ ) then you are fine, and if lower even better. There is no need to get another GnRH agonist or antagonist injection. Repeat the same testosterone measurement monthly. You can expect an upward trend of testosterone, but if there is as well an increase in PSA, then recovery of the receptors in the hypothalamic-pituitary axis (HPA) is occurring and time to reinitiate the $\mathrm{GnRH}$ agonist or antagonist injection. During any IAD interval, "maintenance" with Avodart or Proscar should be considered to continue inhibition of 5AR activity. This allows T to slowly recover but still inhibits the conversion of T to DHT so as to interfere least with the good effects of $T$ on the rest of the body and probably to delay the onset of androgen insensitive prostate cancer (AIPC) clones.

Note these papers regarding the importance of inhibiting androgen/testosterone conversion to dihydrotestosterone: [http://clincancerres.aacrjournals.org/cgi/content/abstract/10/21/7121]. 
"Conclusions: The source of dihydrotestosterone in prostatic tissue after androgen deprivation therapy involves intracrine production within the prostate, converting adrenal androgens to dihydrotestosterone. Dihydrotestosterone still remaining in prostate tissue after androgen deprivation therapy may require new therapies such as treatment with a combination of 5-reductase inhibitors and antiandrogens, as well as castration."

Then there is a study, no longer available by URL that concluded that even with "low dihydrotestosterone" presence, that dihydrotestosterone can influence cancer growth: "Low dihydrotestosterone in cases of aggressive prostate cancer is probably sufficient to activate androgen receptor expression and propagate tumor growth."

Note: Thus all the more reason to "inhibit" this hormone by the use of a 5Alpha Reductase (5AR) inhibitor (dutasteride/ Avodart or finasteride/Proscar).

More from renowned Myers CE, who specializes specifically in the treatment of advanced prostate cancer: "Since I opened my clinic-the American Institute for Diseases of the Prostate in 2002, I've made it a practice to measure dihydrotestosterone levels in each patient we see. And I have to tell you that medical castration, while effective at reducing testosterone from the normal range of $300 \mathrm{ng} / \mathrm{dL}$ to $800 \mathrm{ng} / \mathrm{dL}$ to below $30 \mathrm{ng} /$ $\mathrm{dL}$, often leaves dihydrotestosterone levels within the normal range ( $30 \mathrm{ng} / \mathrm{dL}$ to $80 \mathrm{ng} / \mathrm{dL}$ ). And dihydrotestosterone is ten times more powerful than testosterone at stimulating prostate growth, so a dihydrotestosterone of $30 \mathrm{ng} / \mathrm{dL}$ is potentially as powerful as a testosterone of 300. Dihydrotestosterone formation can be blocked in most patients with either Proscar or Avodart, with Avodart being more consistently effective." In Dr. Myers November 2009 "Prostate Forum" newsletter:

"However, in our clinic, I go further. There are two forms of androgen in men: testosterone and dihydrotestosterone. There is broad agreement that dihydrotestosterone is 10 times more powerful than testosterone in stimulating the growth of prostate cells. There is now also powerful epidemiological, laboratory, and clinical trial data showing that dihydrotestosterone is important in the development and progression of prostate cancer. With this in mind, starting in 1995 I made it a standard practice to also check dihydrotestosterone levels in men on hormonal therapy. I have been surprised by the number of men who have castrate levels of serum testosterone who have serum dihydrotestosterone levels within normal ranges. If dihydrotestosterone is 10 times more powerful, then these men have enough androgen to impair response to hormonal therapy. I think this provides strong support for the use of triple hormonal blockade (addition of Proscar or Avodart) in men with elevated dihydrotestosterone levels. However, I must stress that my views on dihydrotestosterone and the use of Proscar or Avodart are not accepted by many in the prostate cancer field. Nevertheless, I am not the only one who thinks dihydrotestosterone is important. In fact, the major public advocates of this approach are (Medical Oncologists) Stephen Strum and Robert Leibowitz, who have both focused on the development of triple hormonal blockade that includes blockade of dihydrotestosterone production."

\section{Prolactin Level}

Check fasting prolactin level since prolactin SENSITIZES the AR (androgen receptor) and also inhibits Dopamine thus favoring angiogenesis.

From: [http://www.drmyattswellnessclub.com/prostatecancer.htm]

"Prolactin hormone is an additional growth factor to the prostate gland, and rising prolactin levels correlate with progression in advanced prostate cancer cases.

Prolactin receptors are found on prostate cancer cells, and it is postulated that these receptors may facilitate the entry of testosterone into the cell. Even with hormone ablation therapy, detectable androgen remains in the blood from adrenal sources.

Blocking prolactin secretion may therefore be another method for slowing progression of the disease. It is recommended that prolactin levels be kept below 3 in all patients with hormone-responsive cancers."

\section{Medical Oncologist Stephen Strum remarks: "If the fasting} prolactin is 5.0 or higher, start Dostinex (cabergoline) at 0.25 mg every Monday, Wednesday, and Friday. A month later recheck the prolactin level." Dr. Strum provided this additional consideration: "If on cabergoline (Dostinex) and you have been on the drug for over 6 months and the BEP (Basic End Point) of fasting prolactin is $<5.0$, you can stop the cabergoline \& monitor the fasting prolactin. Some patients may never need to go back on cabergoline pending the results of the prolactin level."

Dr. Strum also has this to say regarding ADT: “ADT should be at least ADT with 3 drugs to include anti-androgen (AA) e.g. Ca- 
sodex or Flutamide (Note: or Nilutamide/Nilandron) followed one week later by a GnRH agonist like Lupron or Trelstar or Eligard. I also use Avodart along with the anti-androgen (AA). I monitor the CBC, ultrasensitive PSA, CMP (comprehensive metabolic panel) \& testosterone monthly until stable values. My goal in PSA is $<0.05 \times 12$ months and then off ADT but staying on Avodart. Our published paper used finasteride (Proscar) but dutasteride (Avodart) appears to be a more appropriate 5ARI (5-alpha reductase inhibitor) for higher Gleason score PC." (MY NOTE: The antagonist Firmagon/degarelix does not require an antiandrogen to precede its injection, since this antagonist does not cause a "flare" reaction).

Physicians who ignore these multiple medications that are necessary to block/inhibit androgen/testosterone production and access to the cancer cell have not done their own, personal research and study. They are following what they have been told or what they have read authored by other than Medical Oncologists who specialize specifically in research and treatment of prostate cancer. They do not have the knowledge nor developed the expertise of those Medical Oncologists who have dedicated their lives to specializing specifically in research and treatment of prostate cancer, and more specifically, advanced prostate cancer. These are the Medical Oncologists who absolutely understand the prostate and appropriate treatment of recurring and advanced prostate cancer. Medical Oncologists Strum SS, Myers CE, Leibowitz R, Scholz M, Lam R, Tucker S, Tisman G, to name a few, are in this special category.

Alibhai SM, et al., (2013), a study report in April 2013 brought attention to the importance of Vitamin D in, particularly, the first year of androgen deprivation therapy. [See: http://www. ncbi.nlm.nih.gov/pubmed/23563932]. This study suggests that "Loss of BMD associated with ADT is greatest at the lumbar spine and in the first year. Vitamin D but not calcium may be protective particularly in the first year of ADT use." Accordingly, men moving to ADT should have their 25-hydroxy Vitamin D level determined, and if not well over $50 \mathrm{ng} / \mathrm{ml}$ - preferably within a range of $60 \mathrm{ng} / \mathrm{ml}$ to $75 \mathrm{ng} / \mathrm{ml}$ - it would be important to add Vitamin D3 supplements at a level of around 5000 IU to 6000 IU total daily intake to bring the level up to afford the protection explained above. The 25-hydroxy Vitamin $D$ test should then accompany future PSA level checks at least until the Vitamin D level reaches the preferred range. Strum S, mentioned earlier, has developed a formula called "Dr. Strum's Intensive Bone Formula," available from life extension foundation (LEF). See an explanation of this comprehensive bone formula at the end of this paper.
For an understanding of other areas of treatment for prostate cancer, please visit [http://www.theprostateadvocate.com/ observations.html] and click on any subject.

More provided me in the past by renowned Strum $S$ that should be discussed with your treating physician:

You should be sure that someone is checking your CBC to ensure no significant anemia due to ADT/CHB.

Strum SB, et al., (1997) Anaemia associated with androgen deprivation in patients with prostate cancer receiving combined hormone blockade. Br J Urol 79:933-41, 1997.

Important consideration if experiencing bone issues or to strengthen bone as well as to include in your vitamin intake important vitamins for your general health is the following:

Medical Oncologist Stephen Strum determined a more promising supplement to deal with several PC/health issues, but particularly bone issues, that is available from Life Extension Foundation (LEF) - www.lef.org - known as “Dr. Strum's Intensive Bone Formula." This is, in my opinion, the most comprehensive bone and heart supplement available. Life Extension Foundation (LEF) as item \#01506, this supplement incorporates the several vitamins/products important to bone and heart health. This supplement/formula is explained here:

Dr. Strum's Intensive Bone Formula, See [http://tinyurl.com/ qg2qrwn].

A daily serving of ten capsules includes these nine bonehealthy nutrients:

- Potassium citrate 1173 mg (as potassium citrate supplying 30 milliequivalents of potassium) - favorably affects bone mineral density through a unique calcium-sparing process in the body, increasing the availability of calcium necessary for boneremodeling.

- Extracts of the Ayurvedic herb Cissus quadrangularis $600 \mathrm{mg}$ (aerial part) [std. to $10 \%$ ketosterones $(60 \mathrm{mg})$ ] - scientific research indicates it possesses potent bone health-promoting properties, including support for bone generation, healing, and strengthening capabilities. Very few formulas contain this plant-based bone defender.

- DimaCal ${ }^{\circledR} 800$ mg (as I DimaCal ${ }^{\circledR}$ (Dicalcium Malate) - a patented form of calcium that exhibits significantly better absorption than other calcium supplement options. 
-Vitamin D3 8000 IU - the optimally bioactive form of vitamin D.

- Vitamin K2 200 mcg (as menaquinone-7) - long-acting form essential to transport calcium from the bloodstream into the bone. Working together with vitamin D, it helps maintain youthful calcium musculoskeletal distribution throughout the body.

- Magnesium 400 mg (as magnesium citrate) and Zinc 10 mg (as zinc citrate) - the citrate forms for exceptional bioavailability.

- Boron $6 \mathrm{mg}$ (from boron citrate, aspartate, glycinate) and Silica $5 \mathrm{mg}$ (from standardized Bamboo (Bambusa vulgaris) extract (stem) - to support the bone matrix as it undergoes already-normal growth and turnover.

Other ingredients: vegetable cellulose (capsule), vegetable stearate, silica, maltodextrin. Contains corn.

\section{Dosage and Use}

- Take three capsules in the morning, three capsules in the afternoon, and four capsules in the evening daily with meals, or as recommended by a healthcare practitioner.

\section{Caution}

Individuals consuming more than 2,000 IU/day of vitamin D (from diet and supplements) should obtain a serum 25-hydroxy vitamin D measurement. Do not exceed 10,000 IU per day unless recommended by your healthcare provider. This product is not recommended for individuals with high blood calcium or potassium levels. Because potassium may have numerous drug interactions (most commonly, diuretics and blood pressure medications), consult your healthcare provider before taking this product if you are taking prescription medications and/or have heart or kidney disease. Due to the vitamin $\mathrm{K}$, consult your healthcare provider before taking this product if you are taking anti-platelet or anti-coagulant medications, or have a bleeding disorder.

Therefore, if using Strum's Intensive Bone Formula, though this formula costs around $\$ 56$ per month, when considering that it replaces separate purchases of the vitamins and supplements listed, that price is not as significant, particularly in recognizing you would not likely be needing additional Vit $D$ or Vit $\mathrm{K}$, and the calcium dose is likely sufficient to not require additional calcium supplements; and you would be getting a very comprehensive bone formula. The potassium citrate al- kalinizes urine and helps promote bone formation. You would need to advise your treating physician in order to have your blood serum potassium, blood serum 25-hydroxyvitamin D, blood serum and urine calcium, and parathyroid hormone levels monitored.

There are side effects that accompany androgen deprivation therapy that patients may or may not experience. These side effects and possible remedies are explained in this paper: [http://tinyurl.com/3p9pl3p]

Important also, when moved to androgen deprivation therapy is the paper [http://www.ncbi.nlm.nih.gov/ pubmed/24467669], regarding "Can Supervised Exercise Prevent Treatment Toxicity in Prostate Cancer Patients Initiating Androgen Deprivation Therapy" that concludes "Commencing a supervised exercise program involving aerobic and resistance exercise when initiating ADT significantly reduced treatment toxicity while improving social functioning and mental health. Concurrent prescription of supervised exercise when initiating ADT is therefore advised to minimize morbidity associated with severe hypogonadism."

A patient questioned "I don't know why "supervised" is required, unless a person has no idea of how to exercise moderately." I commented "I expect the idea of "supervised" is because likely a majority of men do not have any idea on how to "appropriately" exercise. Under the supervision of a specialist one would be provided a list of the exercises to perform and equipment available at a facility to accommodate that exercise. Many hospitals have facilities for membership to use their equipment, indoor track, and specialists to guide appropriate exercise. And for those living in cities of moderate size, there are usually one or more such facilities available at moderate cost. I don't know about the "prescribing," since I doubt a health insurer is going to pay for such an exercise program unless considered rehabilitation for a health issue considered requiring rehabilitation. I don't see simply being recommended to exercise as qualifying as rehabilitation. Certainly, for those men who have been involved in programs in the past that included exercises for loosening up, muscle building/toning, winding down, etc. (usually vigorous sports programs or martial arts), they could likely develop their own appropriate exercise program to be performed regularly at home.

The importance, readers, is that an exercise program can alleviate many of the side effects that accompany androgen deprivation therapy, so including this in your therapy is encouraged. 
Should the foregoing ADT show failure and your cancer is showing evidence of becoming hormone refractory with elevating PSA or other diagnostics and your cancer is known to be metastatic, This would be the appropriate time to consider the administration of Provenge/sipuleucel-T to enhance the activity of your immune system so that further medications may be more effective. [See: https://tinyurl.com/ybxm65z3]
Subsequent medications for those with metastatic prostate cancer and prior to having to move to chemotherapy medications can include Zytiga/abiraterone acetate and Xtandi/ enzalutamide. Activity of both explained here: [http://tinyurl. com/9ozr5oa].

Copyright: $\odot 2019$ Maack C. This is an open-access article distributed under the terms of the Creative Commons Attribution License, which permits unrestricted use, distribution, and reproduction in any medium, provided the original author and source are credited. 